\title{
Depression and anxiety in patients with COPD
}

\author{
Abebaw M. Yohannes ${ }^{1}$ and George S. Alexopoulos² \\ Affiliations: ${ }^{1}$ Dept of Health Professions, The Research Institute for Health and Social Care, Manchester \\ Metropolitan University, Manchester, UK. ${ }^{2}$ Weill Cornell Institute of Geriatric Psychiatry, Weill Cornell Medical \\ College, White Plains, NY, USA.
}

Correspondence: Abebaw M. Yohannes, Dept of Health Professions, The Research Institute for Health and Social Care, Manchester Metropolitan University, Manchester, M13 0JA, UK. E-mail: A.yohannesđmmu.ac.uk

ABSTRACT Under-recognised and untreated depression and anxiety symptoms have deleterious effects on physical functioning and social interaction increasing fatigue and healthcare utilisation in patients with chronic obstructive pulmonary disease (COPD). Depression and anxiety are challenging to identify and treat because their symptoms often overlap with those of COPD. The cause(s) of depression and anxiety symptoms are multifactorial and include behavioural, social and biological factors. Less than one-third of COPD patients with comorbid depression or anxiety symptoms are receiving appropriate treatment. Factors that contribute to the lack of provision of treatment are varied, they include patient perceived barriers, for example lack of knowledge and reluctance to receive antidepressant drug therapy; poor treatment compliance and lack of a standardised diagnostic approach; and scarcity of adequate resources for mental health treatment.

The evidence for the efficacy of antidepressant drug therapy in patients with COPD with comorbid depression and anxiety is inconclusive. There are some promising findings regarding pulmonary rehabilitation, psychological therapy and the collaborative care model in reducing depression and anxiety symptoms in patients with COPD, but these findings are limited by short-term follow-up periods. Further work is required to examine the efficacy of these interventions in randomised controlled trials with larger samples and long-term follow-up.

0

@ERSpublications

Untreated comorbid anxiety and depression in patients with COPD have devastating consequences http://ow.ly/vKDYs

\section{Introduction}

During the past two decades, there has been increasing recognition that patients with chronic obstructive pulmonary disease (COPD) with three or more comorbidities are more likely to be frequently hospitalised and may die prematurely compared with COPD patients without comorbidities [1]. Of such comorbidities, anxiety and depression contribute to a substantial burden of COPD-related morbidity, notably by impairing quality of life and reducing adherence to treatment [2]. Untreated and under-recognised depression and anxiety symptoms in patients with COPD have deleterious effects on physical functioning and on social interaction, increasing fatigue and healthcare utilisation $[3,4]$. Depression and anxiety are challenging to identify and treat because their symptoms often overlap with those of COPD [5]. Identifying depression and anxiety, and developing appropriate treatment strategies are critical to improving the quality of life of

Received: Oct 162013 | Accepted after revision: Nov 072013

Support statement: This work was supported by grant numbers P30 MH085943 and R01 MH076829 awarded to G.S. Alexopoulos.

Conflict of interest: Disclosures can be found alongside the online version of this article at err.ersjournals.com

Provenance: Submitted article, peer reviewed

Copyright OERS 2014. ERR articles are open access and distributed under the terms of the Creative Commons Attribution Non-Commercial Licence 4.0. 
COPD patients and reducing their healthcare utilisation. This review synthesises the current understanding of the prevalence and potential mechanism of association, and discusses implications for treatment in patients with COPD with comorbid depression and anxiety symptoms.

\section{What do we know about depression and anxiety?}

Mental health-related disorders are the leading causes of increased disability and impaired quality of life in older people worldwide. Specifically, mood disorders, such as major depression, dysthymias (chronic depressive symptoms of mild severity), minor depression and anxiety disorders (generalised anxiety disorder, phobias and panic disorders) are common in patients with COPD [6, 7]. In a recent longitudinal study by SCHNEIDER et al. [7] of 35000 patients with COPD and with a follow-up of 10 years, the incidence of depression was 16.2 cases per 1000 person-years in the COPD group compared with 9.4 cases per 1000 person-years in the non-COPD control group. In addition, those with severe COPD were twice as likely to develop depression compared with patients with mild COPD [7, 8]. To date, there are no longitudinal studies that have examined the incidence of anxiety disorders in patients with COPD. However, a recent cross-sectional study by EISNER et al. [9] reported that COPD patients are 85\% more likely to develop anxiety disorders compared with healthy, matched controls (controlling for confounding variables such as demographic characteristics and disease severity). In addition, the prevalence of clinical anxiety in COPD outpatients ranges between $13 \%$ and $46 \%$ [5]. Furthermore, COPD patients with comorbid anxiety disorders are twice as likely to exhibit self-reported functional limitations, poorer exercise tolerance and higher frequency of acute exacerbations compared with those without anxiety symptoms. Indeed, anxiety disorders are disabling and, unless adequately treated, they can become chronic, lower selfesteem, predispose to suicidal ideation and increase the risk of hospitalisation $[5,6,8]$.

\section{Mechanism of potential association with COPD}

A recent systematic review and meta-analysis of 25 studies with long-term follow-up revealed that the relationship between COPD and depression is likely to be bidirectional [8], as depression may be both a cause and a consequence of COPD. However, the exact mechanisms linking COPD with depression and anxiety have not been identified. The interrelationship between smoking, depression and/or anxiety and COPD is unclear. Smoking increases the risk and severity of COPD, makes daily activities effortful and stressful, and increases the risk of depression or anxiety in patients with COPD. Associations between anxiety disorders and COPD appear to be largely explained by confounding factors, such as previous history of cigarette smoking and nicotine dependence [10]. However, the relationship of mood disorders to COPD appears to be largely accounted for by nicotine dependence. Thus, these cross-sectional associations do not allow inference about causality but point out the need for specifically designed studies. Depression and anxiety may lead to fear, panic and hopelessness, low self-esteem, social isolation and dependence on caregivers, thereby initiating a vicious circle that perpetuates anxiety and depression.

There is emerging evidence to suggest that low-grade chronic inflammation mediates, in part, the association of depressive symptoms and pulmonary function. Increased inflammatory markers have been documented in both late-life depression [11] and COPD [12]. In a recent study of a population sample of older adults, elevated levels of the inflammatory biomarkers interleukin- 6 and C-reactive protein accounted in part for the association of depressive symptoms with pulmonary obstruction [13]. In addition, there are biological, behavioural and social factors that may contribute to an increase in physical disability and social isolation in patients with COPD (table 1).

A large study examined the prevalence of depression in COPD patients $(n=2118)$, smokers without COPD $(n=335)$ and nonsmokers without COPD $(n=243)$ [14]. The prevalence of depression was $26 \%, 12 \%$ and $7 \%$ in COPD patients, smokers and nonsmokers, respectively. These findings indicate that clinical and biological markers were less important determinants of depression in COPD than disease symptoms and quality of life.

\section{What are the treatment options?}

To date, the available evidence suggests that the effectiveness of treatment of depression or anxiety using selective serotonin reuptake inhibitors (SSRIs) in patients with COPD is questionable. This is partly due to patients' fear that antidepressant drugs are addictive and have potential side-effects, and perceived stigma associated with depression. In addition, lack of adequate support and explanation of depression by the healthcare professionals of the reasons for and the efficacy of treatment leads patients to decline this valuable treatment. The collaborative care model (case management) of partnership with patients and family has been shown to be beneficial in the treatment of depression in patients with chronic diseases [15]. However, its efficacy in COPD patients with comorbid anxiety or depression is unknown. A recent Cochrane review [16] examined studies that investigated the benefits of pharmacological interventions for 
TABLE 1 Association, symptoms, impact and treatment options for depression and anxiety in chronic obstructive pulmonary disease

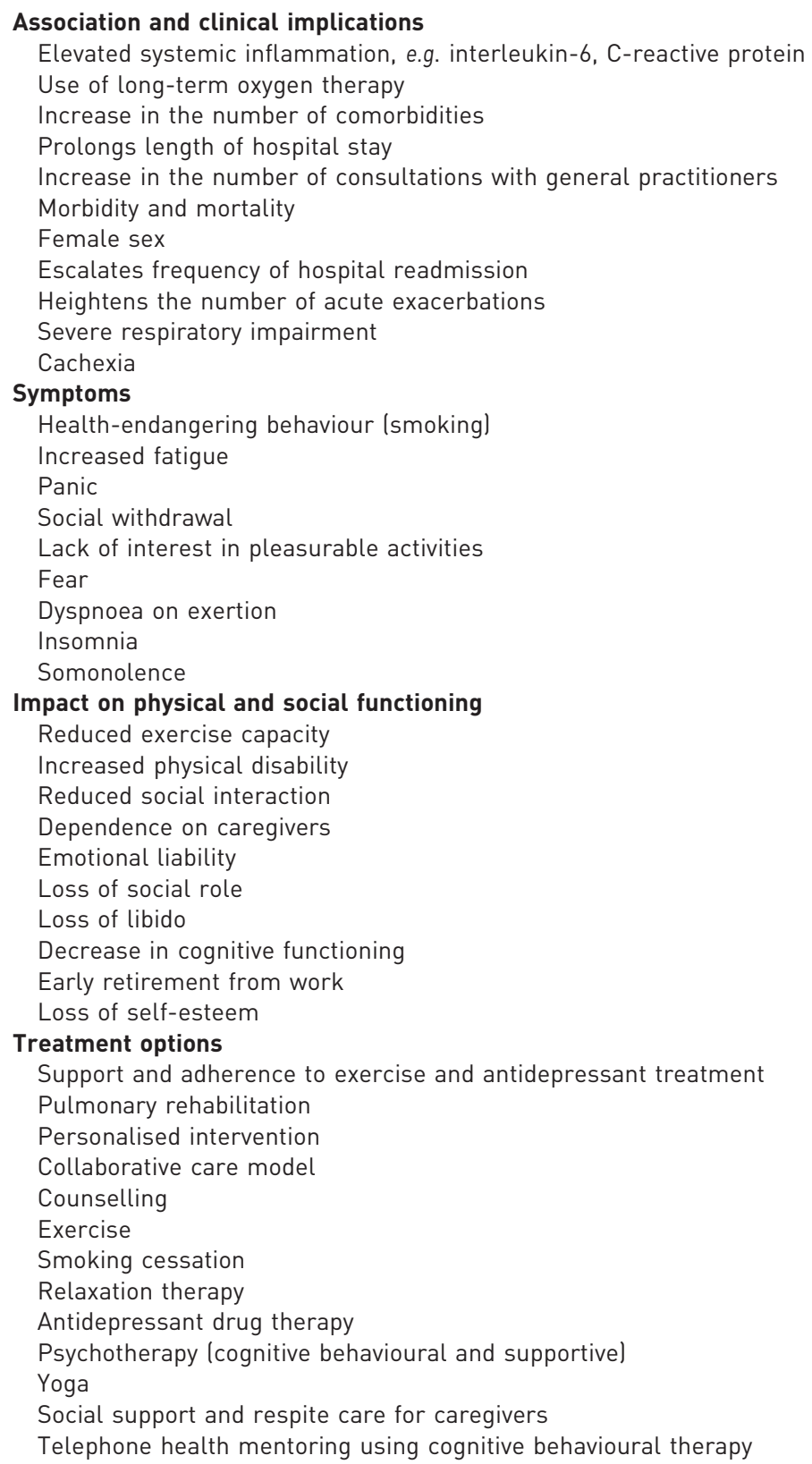

treatment anxiety in patients with COPD. Their findings indicate that studies were heterogeneous and treatment efficacy was inconclusive. Therefore, well-controlled randomised trials are needed. Recently, a personalised nine-session intervention for depression and COPD (PID-C) was developed for patients with major depression and severe COPD [17]. PID-C is offered by care managers who, through support and targeted interventions, help patients to work on their exercise regimens and take antidepressants. The care managers also collaborate with the patients' physicians in monitoring the patients' treatment and progress. A randomised controlled trial showed that PID-C led to a higher remission rate of depression and greater reduction in depressive symptoms in dyspnoea-related disability than usual care over 28 weeks and 6 months after the last session [18]. Low severity of dyspnoea-related disability and adherence to antidepressants predicted subsequent improvement of depression. Exercise and low depression severity predicted improvement of dyspnoea-related disability. 
A recent systematic review investigated the efficacy of comprehensive pulmonary rehabilitation (exercise plus education) in patients with COPD patients and showed that reduction in levels of depressive and anxiety symptoms in the short-term was comparable with usual care [19]. In addition, a recent uncontrolled intensive 3 -week outpatient pulmonary rehabilitation programme ( $6 \mathrm{~h}$ per day for 5 days per week) showed significant improvement in depression and anxiety in patients with COPD [20]. Again, the long-term benefits and their clinical significance require further investigation. Acute inpatient rehabilitation was followed by improvement of depressive symptoms and disability, even in older patients with severe COPD and major depression. Improvement of depression was unrelated to the use of antidepressant drugs and was attributed to the behavioural interventions of pulmonary rehabilitation [21]. However, the long-term benefit of pulmonary rehabilitation in reducing anxiety and depression is unknown. In addition, further work is required on the efficacy of maintenance therapy to alleviate these symptoms and achieve full remission.

There is some evidence to suggest that psychological therapy, including cognitive behavioural therapy and counselling, may improve depressive and anxiety symptoms in patients with COPD $[5,6]$. However, there is currently uncertainty over the "dosage" and the duration of therapy, even for mild cases of anxiety and depression. In addition, there is limited availability of psychological therapy in primary care settings for this patient group. It is worth considering making psychological therapy resources available using web-based technology as a supplement therapy.

WALTERS et al. [22] examined the efficacy of telephone health mentoring using cognitive behavioural therapy $(\mathrm{n}=80)$ versus usual care $(\mathrm{n}=74)$ in a 12 -month treatment programme. The experimental group received 16 30-min telephone calls over 12 months, with increasing time between calls. The control group received the usual care provided by their general practitioners and a monthly phone call by a research nurse. Anxiety decreased in both groups at 12 months. Health mentoring improved the capacity for self-management, but the two groups had similar scores for quality of life at the end of the treatment phase. Moreover, there was no difference in depression scores between the two groups. BUCKNALL et al. [23] reported that a minority of COPD patients who could learn to implement self-management effectively were younger and were more likely to be living with others. These patients had a significantly reduced risk of COPD readmission.

\section{Barriers to treatment of interventions}

The available evidence suggests that less than one-third of COPD patients with comorbid depression or anxiety are receiving appropriate treatment for this. Factors that contribute to the lack of provision of treatment are multifactorial. In their elegant review, MAURER et al. [6] reported the multistage barriers for detection and treatment of anxiety and depression in patients with COPD. These include 1) patientperceived barriers, for example lack of knowledge and reluctance to disclose symptoms of anxiety or depression; 2) physician-perceived barriers, for example lack of a standardised diagnostic approach for anxiety and depression, short-consultation time and lack of confidence to pursue in-depth psychological assessment; and 3) system-level barriers, for example poor communication between primary care and mental health systems, and lack of adequate resources for mental health treatment. In order to address these barriers, an integrated treatment approach is required from healthcare professionals, patients and caregivers. In addition, the healthcare providers should be ready to provide appropriate resources to improve the quality of service provision and clinical practice.

Current screening tools for anxiety and depression in patients with COPD have primarily been validated for patients with other chronic diseases. The Hospital Anxiety Depression and the Beck Depression and Anxiety Inventory scales have been recommended as the preferable screening tools for anxiety and depression in patients with COPD [6]. However, some of the items in these scales contain somatic symptoms, which make it difficult to decipher because of the overlap symptoms of COPD and depression or anxiety. Thus, designing disease-specific anxiety and depression scales for patients with COPD is a worthy future endeavour.

\section{Challenges for research and clinical practice}

There is little evidence to suggest whether routine screening (which is resource intensive) may improve treatment for anxiety and depression in patients with COPD. Therefore, healthcare professionals should play an active role, at least for those identified with clinical depression and anxiety, to ensure appropriate treatment and to monitor its efficacy. Such patients also present enormous challenges to families, carers and social care and social support networks.

However, the lack of strong evidence for the efficacy of antidepressant drug therapy in patients with COPD with comorbid depression and anxiety necessitates well-controlled clinical trials to explore efficacy of antidepressant drug therapy in inducing sustained remission. In addition, there is very little evidence to show to healthcare providers (e.g. physicians and nurses) and funders (e.g. health commissioners) that treatment is cost-effective and/or reduces healthcare utilisation. 
At the moment, pulmonary rehabilitation programmes do not provide special provision for COPD patients with clinically significant anxiety and depression. Interventions that are specifically targeted (by design) to the severity of respiratory impairment, sex, culture and duration of illness may have impact in terms of prognosis and are worthy of investigation.

\section{Conclusion}

Untreated comorbid anxiety and depression in patients with COPD have devastating consequences, overwhelm the coping strategies of COPD patients and their caregivers, and may increase healthcare utilisation. There are some promising findings regarding pulmonary rehabilitation, smoking cessation, and psychological and antidepressant drug therapy in reducing anxiety and depressive symptoms in patients with COPD. However, these findings require further testing to examine their efficacy in well-controlled randomised controlled trials with larger samples and long-term follow-up.

\section{References}

1 Sode BF, Dahl M, Nordestgaard BG. Myocardial infarction and other comorbidities with chronic obstructive pulmonary disease: a Danish Nationwide study of 7.4 million individuals. Eur Heart J 2011; 32: 2365-2375.

2 Yohannes AM, Willgoss TG, Baldwin RC, et al. Depression and anxiety in chronic heart failure and chronic obstructive pulmonary disease: prevalence, relevance, clinical implication and management principles. Int J Geriatr Psychiatry 2010; 25: 1209-1221.

3 Doyle T, Palmer S, Johnson J, et al. Association of anxiety and depression with pulmonary-specific symptoms in chronic obstructive pulmonary disease. Int J Psychiatry Med 2013; 45: 189-202.

4 Dalal AA, Shah M, Lunacsek O, et al. Clinical and economic burden of depression/anxiety in chronic obstructive pulmonary disease patients within a managed care population. COPD 2011; 8: 293-299.

5 Willgoss TG, Yohannes AM. Anxiety disorders in patients with COPD: a systematic review. Respir Care 2013; 58: 858-866.

6 Maurer J, Rebbapragada V, Borson S, et al. Anxiety and depression in COPD: current understanding, unanswered questions, and research needs. Chest 2008; 134: 43S-56S.

Schneider C, Jick SS, Bothner U, et al. COPD and the risk of depression. Chest 2010; 137: Suppl. 4, 341-347.

8 Atlantis E, Fahey P, Cochrane B, et al. Bidirectional associations between clinically relevant depression or anxiety and COPD: a systematic review and meta-analysis. Chest 2013; 144: 766-777.

9 Eisner MD, Blanc PD, Yelin EH, et al. Influence of anxiety on health outcomes in COPD. Thorax 2010; 65: 229-234.

10 Goodwin RD, Lavoie KL, Lemeshow AR, et al. Depression, anxiety, and COPD: the unexamined role of nicotine dependence. Nicotine Tob Res 2012; 14: 176-183.

11 Alexopoulos GS, Morimoto SS. The inflammation hypothesis in geriatric depression. Int J Geriatr Psychiatry 2011; 26: 1109-1118.

12 Huerta A, Crisafulli E, Menéndez R, et al. Pneumonic and non-pneumonic exacerbations of COPD: inflammatory response and clinical characteristics. Chest 2013; 144: 1134-1142.

13 Lu Y, Feng L, Feng L, et al. Systemic inflammation, depression and obstructive pulmonary function: a populationbased study. Respir Res 2013; 14: 53.

14 Hanania NA, Müllerova H, Locantore NW, et al. Determinants of depression in the ECLIPSE chronic obstructive pulmonary disease cohort. Am J Respir Crit Care Med 2011; 183: 604-611.

15 Katon WJ, Lin EHB, Von Korff M, et al. Collaborative care for patients with depression and chronic illnesses. N Engl J Med 2010; 363: 2611-2620.

16 Usamani ZA, Carson KV, Cheng JN, et al. Pharmacological interventions for the treatment of anxiety disorders in chronic obstructive pulmonary disease. Cochrane Database Syst Rev 2011; 11: CD008483.

17 Alexopoulos GS, Raue PJ, Sirey JA, et al. Developing an intervention for depressed, chronically medically ill elders: a model from COPD. Int J Geriatr Psychiatry 2008; 23: 447-453.

18 Alexopoulos GS, Kiosses DN, Sirey JA, et al. Personalised intervention for people with depression and severe COPD. Br J Psychiatry 2013; 202: 235-236.

19 Coventry PA, Hind D. Comprehensive pulmonary rehabilitation for anxiety and depression in adults with chronic obstructive pulmonary disease: systematic review and meta-analysis. J Psychsom Res 2007; 63: 551-565.

20 Von Leupoldt A, Taube K, Lehmann K, et al. The impact of anxiety and depression on outcomes of pulmonary rehabilitation in patients with COPD. Chest 2011; 140: 730-736.

21 Alexopoulos GS, Sirey JA, Raue PJ, et al. Outcomes of depressed patients undergoing inpatient pulmonary rehabilitation. Am J Geriatr Psychiatry 2006; 14: 466-475.

22 Walters J, Cameron-Tucker H, Wills K, et al. Effects of telephone health mentoring in community-recruited chronic obstructive pulmonary disease on self-management capacity, quality of life and psychological morbidity: a randomised controlled trial. BMJ Open 2013; 3: e003097.

23 Bucknall CE, Miller G, Lloyd SM, et al. Glasgow supported self-management trial (GSuST) for patients with moderate to severe COPD: randomised controlled trial. BMJ 2012; 344: e1060. 